\title{
Quantitation of cerebrospinal fluid lactic acid in infectious and non-infectious neurological diseases
}

\author{
Sérgio Monteiro de Almeida ${ }^{1,3}$, , Fernanda \\ Luize Faria', Karina de Goes Fontes ${ }^{2}$, Gisele \\ Maria Buczenko ${ }^{3}$, Denize Bonato Berto ${ }^{4}$, Sonia \\ Mara Raboni ${ }^{1}$, Luine Rosele Vidal ${ }^{1}$ and Meri \\ Bordignon Nogueira ${ }^{1}$ \\ ${ }^{1}$ Virology Section, Clinical Pathology Laboratory, \\ Hospital de Clínicas, Federal University of Paraná, \\ Curitiba, PR, Brazil \\ 2 Biochemistry Section, Clinical Pathology \\ Laboratory, Hospital de Clínicas, Federal University \\ of Paraná, Curitiba, PR, Brazil \\ ${ }^{3}$ CSF Section, Clinical Pathology Laboratory, \\ Hospital de Clínicas, Federal University of Paraná, \\ Curitiba, PR, Brazil \\ ${ }^{4}$ Laboratório Central do estado do Paraná, Curitiba, \\ PR, Brazil (LACEN)
}

\begin{abstract}
Background: The differential diagnosis between bacterial and viral meningitis is not easy in some cases. Cerebrospinal fluid (CSF) analysis is essential for establishing this diagnosis. The objectives were to quantitate lactic acid (LA) concentrations in bacterial and viral meningitis, and other central nervous system (CNS) diseases in order to evaluate the diagnostic utility of CSF LA for discriminating bacterial from viral meningitis.

Methods: CSF LA was measured in 139 CSF samples from seven groups: viral meningitis with classic CSF; suspicion of viral meningitis with neutrophils in CSF; bacterial meningitis; non-infectious neurological diseases; chronic meningitis; traumatic lumbar puncture (LP) and normal CSF.

Results: CSF LA was higher in bacterial meningitis $8.7+5.4 \mathrm{mmol} / \mathrm{L}$ compared with viral meningitis $(1.9+0.6)$ and the other groups $(p<0.0001)$. CSF LA in the groups with viral meningitis was not different compared to groups with non-infectious CNS diseases and chronic meningitis. The ability of CSF LA to discriminate bacterial from viral meningitis showed a sensitivity of $80 \%$ and specificity of $97 \%$, positive predictive value (PPV) of $94 \%$, and negative predictive value (NPV) of $89 \%$.

Conclusions: CSF LA is a powerful test to discriminate bacterial from viral meningitis with high sensitivity,

\footnotetext{
*Corresponding author: Sérgio Monteiro de Almeida, MD, PhD, Hospital de Clínicas - UFPR, Seção de Virologia,

Setor Análises Clínicas, Rua Padre Camargo 280, 80060-240 Curitiba, PR, Brazil

Phone/Fax: +55 (41) 3360-7974,

E-mail: sergio.ma@pop.com.br

Received November 11, 2008; accepted March 24, 2009
}

specificity and predictive values. CSF LA can help in the cases with diagnostic uncertainty.

Clin Chem Lab Med 2009;47:755-61.

Keywords: bacterial meningitis; cerebrospinal fluid (CSF); lactic acid (LA); lymphocytic meningitis; neurological diseases; viral meningitis.

\section{Introduction}

The differential diagnosis between bacterial and viral meningitis is crucial for treatment and prognosis and the clinical presentation is not always clear. Bacterial meningitis is a life-threatening disease with high mortality and neurologic sequelae. However, it is a treatable disease and early antibacterial therapy is essential for a good outcome. Viral meningitis usually subsides spontaneously. The differential diagnosis is normally based on cellular characteristics of cerebrospinal fluid (CSF), total white blood cells (WBC) count and cell type, and biochemical characteristics, specifically glucose $(\mathrm{GL})$. In some cases the diagnosis is difficult. For example, partially treated bacterial meningitis could present with different cell patterns and the classic bacteriological diagnostic methods, cultures and Gram stain smear usually are negative. CSF characteristics can resemble viral meningitis in immunosuppressed patients. Viral meningitis, especially enterovirus, can present with predominance of neutrophils during the first $6 \mathrm{~h}$ of infection, with normal CSF glucose concentrations. CSF glucose is used as the classic test to differentiate between bacterial and viral meningitis. However, in some cases, blood glucose is not available at the time that CSF is obtained. CSF glucose is correlated with blood glucose concentrations (1-4).

The etiology of acute infectious meningitis is bacterial or viral. After the first month of life, bacteria responsible for $70 \%-90 \%$ of cases of acute bacterial meningitis in most regions of the world are N. meningitidis and $S$. pneumoniae $(5,6)$. The incidence of infection by $H$. influenzae showed significant decline following systematic vaccination. On the other hand, viral meningitis is a global disease that can be sporadic or epidemic. Despite the low mortality rates, these can have high morbidity (7). Non-polio enteroviruses are responsible for the majority of cases of viral meningitis, $50 \%-80 \%$, especially, during the summer.

CSF lactic acid (LA) is considered a good biomarker for differentiating bacterial from viral meningitis at a cut-off of $3.5 \mathrm{mmol} / \mathrm{L}$ (8-12).

The CSF LA concentration in LA is directly dependent upon its production rate in the brain. There is sub- 
stantial evidence, in patients and animals, that blood and CSF lactate concentrations are largely independent of each other. Thus, intravenous infusion of LA in dogs was sufficient to raise the blood concentration 6-fold, yet, failed to increase CSF concentrations (13). This is important in clinical practice because it is not necessary to collect matched plasma. CSF lactate concentrations are also useful for the diagnosis of post surgical acute bacterial meningitis, where there is not an increase in specific cells or proteins $(2,14)$.

The aims of this study were: to analyze LA concentrations in bacterial and viral meningitis compared to other central nervous system (CNS) diseases with infectious and non-infectious etiology; to calculate the diagnostic characteristics of CSF LA for the differential diagnosis of acute bacterial and viral meningitis; to determine CSF LA concentrations in cases with suspect viral meningitis but with a predominant increase in CSF neutrophils, and to determine the influence of red blood cells (RBCs) in CSF, as a result of traumatic lumbar puncture (LP), on CSF LA concentrations.

\section{Materials and methods}

A prospective longitudinal study was conducted by the Clinical Pathology Laboratory of the Hospital de Clínicas, Universidade Federal do Paraná (HC-UFPR). This study was approved by the HC-UFPR Institutional Reviews Board.

All CSF samples were collected by LP. CSF and plasma LA was determined, using the dimension AR machine (Dade Behring, Deerfield, IL, USA).

CSF total protein (TP) was measured using a turbidimetric method with sulphosalicylic acid. CSF glucose was measured using an enzymatic method. CSF total cell count (WBCs) was performed using a Fuchs Rosenthal chamber. A WBC count $>4$ cells $/ \mathrm{mm}^{3}$ was considered to be increased. For the differential cell count, CSF samples were concentrated by Shandon Cytospin (Pittsburgh, USA) and the slides stained using the May Grünwald-Giemsa technique. A predominance of cells was considered when they were $>50 \%$.

A total of 139 CSF samples were studied. The mean (SD) age was 21 years (20), with 53\% males. The samples were distributed into the following groups:

Group $1(n=33)$ : CSF samples with characteristics of viral meningitis: increased numbers of WBCs with a predominance of lymphocytes and glucose $>50 \mathrm{mg} / \mathrm{dL}$ $(\times 0.0555=\mathrm{mmol} / \mathrm{L})$. Polymerase chain reaction $(P C R)$ for enterovirus, herpes simplex virus (HSV)-1, HSV-2, citomegalovirus (CMV), Epstein-Barr virus (EBV), varicella-zoster virus (VZV), human herpes virus (HHV)-8, HHV-6A, HHV-6B, HHV-7 (15) was performed in 15 samples. One sample was positive for enterovirus.

Group $2(n=8)$ : CSF samples from patients with a clinical suspicion of viral meningitis, with increased numbers of WBCs, and a predominance of neutrophils and glucose $>50 \mathrm{mg} / \mathrm{dL}$. PCR for the same viruses searched for in group 1 was performed on five samples; all were negative.

Group $3(n=20)$ : CSF samples with characteristics of untreated acute bacterial meningitis: increased numbers of WBCs with a predominance of neutrophils, glucose $<50$ $\mathrm{mg} / \mathrm{dL}$. Bacteria identified by Gram smear or culture: $N$. meningitidis $B$ (3 samples); $N$. meningitidis $C$ (2 samples); Streptococcus pneumoniae (2 samples); Enterobacter cloa- cae (2 samples); Acinetobacter sp.; Enterococcus faecium; S. aureus; Streptococcus $D$ and not identified (7 samples).

Group $4(n=4)$ : CSF samples from patients with suspicion of chronic meningitis, Mycobacterium tuberculosis $(n=1)$; fungus ( 2 cases with Cryptococcus neoformans and one case with candida sp.).

Group $5(n=17)$ : CSF samples from patients with noninfectious neurological diseases: mitochondrial disorders $(n=4)$; motor neuron disease $(n=3)$; encephalopathy $(n=2)$; dementia $(n=2)$; ataxia $(n=2)$; paraparesis $(n=1)$; myasthenia (1); leukodystrophy $(n=1)$; optic neuromyelitis $(n=1)$.

Group $6(n=42)$ : control group; CSF samples from patients with clinical suspicion of acute meningitis, but WBC count, TP and glucose being normal. Bacteria were not isolated by culture or Gram stain. Red blood cells (RBCs) mean+DP. $0.8+1.1$; median 0.3.

Group $7(n=15)$ : CSF samples from patients with suspicion of acute meningitis, and normal WBC count, TP and glucose. However, there was a large number of RBCs in the CSF due to traumatic LP. This group was included with the intention of evaluating the interference effect of RBCs with determination of CSF lactate. RBCs mean + DP; $6105+17,372$; median 355 .

CSF biochemistry and cell characteristics of all groups studied are shown in Table 1.

\section{Statistical analysis}

Continuous variables were compared using non-parametric testing comparing all the five groups with the Kruskal-Wallis test. The groups were compared, two by two, using the Mann-Whitney test. A $p<0.05$ was used for statistical significance. The results were presented as mean (SD).

To evaluate the characteristics (16) of CSF LA quantitation for the diagnosis of acute untreated bacterial meningitis and viral meningitis, the following formulas were used: sensitivity: $[\mathrm{TrP} /(\mathrm{TrP}+\mathrm{FN})] \times 100 ;$ specificity: $[\mathrm{TN} /(\mathrm{TN}+\mathrm{FP})] \times 100$; positive predictive value (PPV): $[\mathrm{TrP} /(\mathrm{TrP}+\mathrm{FP})] \times 100$; negative predictive value (NPV): $[\mathrm{TN} /(\mathrm{TN}+\mathrm{FN})] \times 100$; detection rate: TrP/total tested; Youden index: [(sensitivity + specificity)-1]; error ratio: $(\mathrm{FP}+\mathrm{FN}) / \mathrm{TrP}$; combined error: $(\mathrm{FP}+\mathrm{FN}) /$ total tested. [True positive (TrP); true negative (TN); false positive (FP); false negative (FN)]. The CSF LA cut-off threshold used was $3.5 \mathrm{mmol} / \mathrm{L}$.

\section{Results}

CSF LA concentrations were higher in the group with acute bacterial meningitis, mean (SD) $[8.7$ (5.4) $\mathrm{mmol} / \mathrm{L}]$, than in the groups with viral meningitis, with normal CSF, non-infectious CNS diseases and chronic meningitis [1.9 (0.6), 1.8 (0.6), 3.1 (3.7), 5.5 (2.8) $\mathrm{mmol} / \mathrm{L}$, respectively], $\mathrm{p}<0.0001$ (Table 2 and Figure 1). CSF LA concentrations in the group with chronic meningitis (group 4) was less, although not statistically significant, than concentrations seen in patients with acute bacterial meningitis $(p=0.13)$. CSF LA concentrations in the group with chronic meningitis were higher, but not statistically significant, than values seen in groups $1,2,5$ and $6(p=0.088,0.124$, $0.208,0.080$, respectively).

In five cases, LA was measured in CSF and blood matched samples. For two cases from the group with untreated bacterial meningitis, CSF LA was 9.0 and 


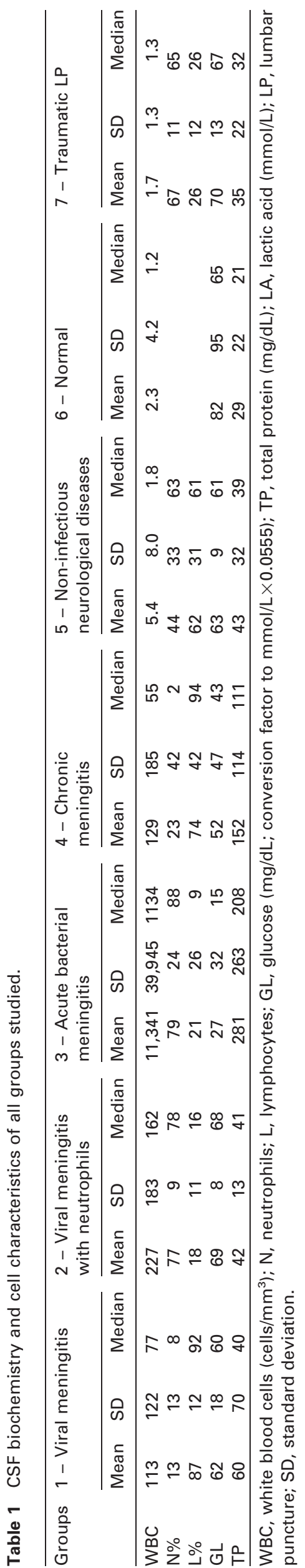

$2.3 \mathrm{mmol} / \mathrm{L}$ and blood LA was 0.3 and $0.5 \mathrm{mmol} / \mathrm{L}$, respectively. For three cases from groups $5,6,7, \mathrm{CSF}$ LA was $2.4,3.6$ and $2.0 \mathrm{mmol} / \mathrm{L}$ and blood LA was 2.8, 1.7 and $1.1 \mathrm{mmol} / \mathrm{L}$, respectively. CSF LA mean (SD) was 3.9 (2.9) with a median of $2.4 \mathrm{mmol} / \mathrm{L}$. This was higher compared with blood where the mean (SD) was $1.3(1.0)$ and the median was $1.1 \mathrm{mmol} / \mathrm{L}$. The Spearman correlation coefficient $(-0.2)$ indicated that there was no correlation between CSF and blood LA concentrations. CSF values were similar to values in serum in cases where the CSF LA was low. In cases of untreated bacterial meningitis, the CSF LA values were 5-30 times higher than serum LA values, indicating intrathecal LA production that was independent of blood.

In the group of patients with non-infectious neurological diseases (group 5), four patients had mitochondrial diseases; two with CSF LA values $>3.5$ $\mathrm{mmol} / \mathrm{L}(4.3$ and $6.6 \mathrm{mmol} / \mathrm{L})$ and two had CSF LA $<3.5 \mathrm{mmol} / \mathrm{L}$ (1.8 and $2.2 \mathrm{mmol} / \mathrm{L}$ ).

In order to evaluate the interference effect of RBCs on measured CSF LA due to traumatic LP, we compared the CSF LA values in group 6 and group 7. RBC values from group 6 , mean+DP; $0.8+1.1$; median 0.3 ; CSF LA was $1.8(0.6) \mathrm{mmol} / \mathrm{L}$. RBC values from group 7, mean+DP; $6105+17,372$; median 355; CSF LA was 1.7 (0.5) $\mathrm{mmol} / \mathrm{L}(\mathrm{p}=0.1734)$ Table 2.

The diagnostic characteristics of CSF LA, using a cut-off of $3.5 \mathrm{mmol} / \mathrm{L}$, are shown in Table 3. Use of CSF LA for discriminating bacterial meningitis from viral meningitis, normal CSF, other non-infectious CNS diseases and chronic meningitis showed a sensitivity of $80 \%$ and a specificity of $93 \%$. When comparing bacterial meningitis to viral meningitis (with typical CSF characteristics) specificity increased to $97 \%$. Comparing bacterial meningitis to suspected viral meningitis (with neutrophils in the CSF), sensitivity, specificity, and PPV were high, but NPV decreased $(67 \%)$.

\section{Discussion}

In this study, CSF LA concentrations were significantly higher (4.6 times) in the group with bacterial meningitis compared with the group with viral meningitis. In the group with viral meningitis, CSF LA concentrations were similar to the control group.

In the group with suspected viral meningitis but with a predominance of neutrophils in the CSF, CSF LA was 2.2 times higher than in the group with classic viral meningitis, but lower than the group with untreated bacterial meningitis. CSF from patients with viral meningitis can have a predominance of neutrophils during the first $6 \mathrm{~h}$ of the disease, with CSF glucose concentrations and the CSF/serum glucose ratio being normal. This is more common among patients with meningoencephalitis caused by enterovirus. CSF LA showed lower concentrations in this group compared to the group with bacterial meningitis. This suggests that in those with viral meningitis, but with a predom- 
Table 2 CSF lactic acid ( $\mathrm{mmol} / \mathrm{L}$ ) concentration in the groups studied.

\begin{tabular}{|c|c|c|c|}
\hline \multirow[t]{2}{*}{ Groups } & \multicolumn{3}{|c|}{ CSF lactic acid, $\mathrm{mmol} / \mathrm{L}$} \\
\hline & Mean & $\pm S D$ & Median \\
\hline Viral meningitis & 1.9 & 0.6 & 1.9 \\
\hline Viral meningitis with neutrophils & 2.5 & 0.4 & 2.4 \\
\hline Acute bacterial meningitis & 8.7 & 5.4 & 7.3 \\
\hline Chronic meningitis & 5.5 & 2.8 & 5.0 \\
\hline Non-infectious neurologic diseases & 3.1 & 3.7 & 1.9 \\
\hline Normal & 1.8 & 0.6 & 1.7 \\
\hline CSF with traumatic LP & 1.7 & 0.5 & 1.5 \\
\hline
\end{tabular}

LP, lumbar puncture; SD, standard deviation.

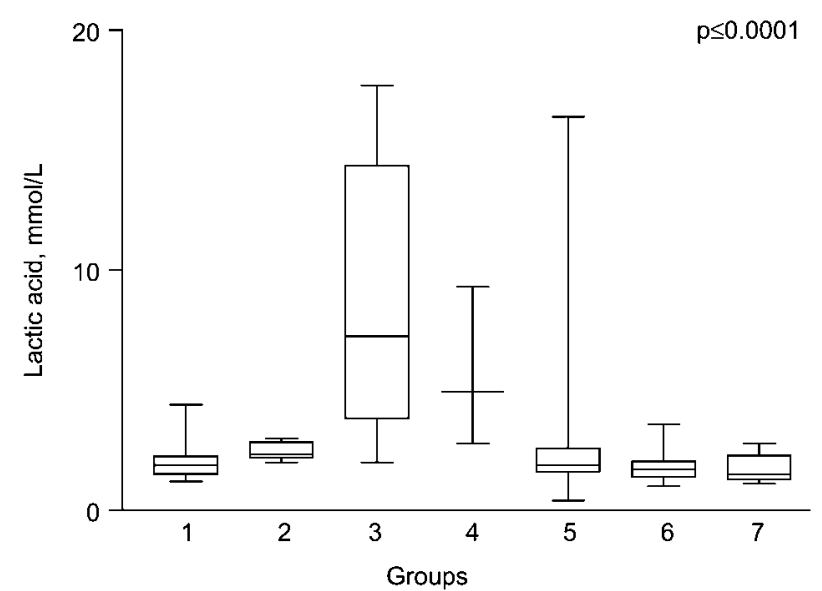

Figure 1 CSF lactic acid ( $\mathrm{mmol} / \mathrm{L}$ ) concentration in the studied groups (results median \pm interquartiles).

inance of neutrophils, CSF LA can help discriminate viral meningitis from acute bacterial meningitis.

Non-polio enteroviruses are the main etiologic agent for viral meningitis. Among the enterovirus group, there is an important division into the Picornaviridae family: Echovirus, Poliovirus and the Coxsackievirus of the groups $A$ and $B$. Enterovirus numbers 70 and 71, which show a strong neurotropism, are associated with meningoencephalitis, poliolike paralytic syndromes, Guillain Barré Syndrome and meningitis. Coxsackievirus sub-group $B$ is responsible for $60 \%$ of meningitis among children $<3$ years of age $(7,17)$. Herpes family viruses are responsible for $4 \%$ of meningitis cases. Meningitis is more frequently caused by HSV-2, HSV-1, 2 and EBV, and is associated with recurrent lymphocytic meningitis $(7,18)$. Almost $5 \%-10 \%$ of HIV positive patients have meningitis which can occur during any phase of the infection; although occurring more frequently during seroconversion.

Most efforts have tried to establish tests that discriminate bacterial meningitis from viral meningitis. This is because bacterial meningitis is a life-threatening disease and has a much poorer prognosis compared with viral meningitis. Since bacterial meningitis can be cured successfully in the early phase of the disease, tests are needed to diagnose bacterial meningitis accurately and reliable with high-sensitivity and specificity (12). Lactate concentrations are particularly important when the CSF Gram stain is negative and there is a predominance of polymorphonuclear cells and low CSF glucose concentrations $(2,14)$.

There has been interest in the diagnostic value of increased CSF lactate for the identification of bacterial meningitis and partially treated bacterial meningitis. This is in contrast to the usual findings of normal lactate concentrations in viral meningitis (19-27). CSF lactate concentrations are also increased in fungal meningitis. In partially treated meningitis, intermediate lactate concentrations have been reported. The decrease in lactate has been considered indicative of

Table 3 Diagnostic characteristics of CSF lactate for differential diagnosis of acute bacterial meningitis (LA limit of 3.5 $\mathrm{mmol} / \mathrm{L})$.

\begin{tabular}{|c|c|c|c|c|}
\hline & $\begin{array}{l}\text { Viral meningitis } \\
\text { compared with } \\
\text { groups } 3,4,5,6\end{array}$ & $\begin{array}{l}\text { Bacterial meningitis } \\
\text { compared with } \\
\text { groups } 1,4,5,6\end{array}$ & $\begin{array}{l}\text { Bacterial } \\
\text { compared with } \\
\text { viral meningitis }\end{array}$ & $\begin{array}{l}\text { Bacterial compared } \\
\text { with viral meningitis } \\
\text { with neutrophils }\end{array}$ \\
\hline Total tested & 116 & 116 & 53 & 28 \\
\hline TrP & 32 & 16 & 16 & 16 \\
\hline Sensitivity, \% & 96.96 & 80 & 80 & 80 \\
\hline Specificity, \% & 26.5 & 92.71 & 96.96 & 100 \\
\hline PPV, \% & 34.4 & 69.57 & 94.12 & 100 \\
\hline NPV, \% & 95.65 & 95.70 & 88.89 & 66.67 \\
\hline Efficiency, \% & 46.55 & 90.52 & 90.57 & 85.71 \\
\hline Detection rate, \% & 27.59 & 14 & 30 & 57.14 \\
\hline Youden index & 0.23 & 0.73 & 0.77 & 0.8 \\
\hline Error ratio & 1.94 & 0.69 & 0.31 & 0.25 \\
\hline Combined error & 0.53 & 0.09 & 0.09 & 0.14 \\
\hline
\end{tabular}

$\mathrm{TrP}$, true positives; PPV, positive predictive value; NPV, negative predictive value. 
effective medication and resolution of infection (28, 29). Infectious chronic meningitis can be seen in conjunction with tuberculosis, syphilis, fungus (mainly $C$. neoformans), cysticercosis, and histoplasmosis (30). Some investigators describe overlapping CSF lactate concentrations in viral, partially treated bacterial meningitis and tuberculosis meningitis. This limits the value of the marker for differentiating these disorders (21). In our study, CSF lactate was higher in acute untreated bacterial meningitis compared with meningitis, although the difference was not statistically significant. These findings should be confirmed due to the small number of samples from patients with chronic meningitis.

The occurrence of increased CSF lactate concentrations have also been described in acute cerebral infarction, reflecting tissue acidosis and increased anaerobic glycolysis (31). Elevated lactate has also been reported in cerebral hemorrhage (32). Another issue we studied was whether the presence of RBCs in the CSF, as result of a traumatic LP, could interfere with CSF lactate concentrations. We found no difference between this groups and control patients.

The non-significant Spearman correlation coefficient in the matched CSF and blood samples suggests that there is no correlation between CSF and blood LA. In cases with low CSF LA, the values are similar to values in serum. In the cases with untreated bacterial meningitis, the CSF LA values are 5-30 times higher than that seen in serum. The higher values of LA in CSF compared with paired blood samples, found in this study, indicate independent intrathecal production. The concentration of LA in brain is dependent directly upon its rate of production. There is evidence, from patients and animal studies, that blood and CSF lactate concentrations are largely independent of each other $(13,33)$. This is a very important characteristic of LA and offers an advantage over CSF glucose determination since CSF glucose is related to blood glucose.

The normal range for lumbar CSF lactate is usually reported as being between 10 and $20 \mathrm{mg} / \mathrm{dL}$ (1.1-2.2 $\mathrm{mEq}$ or $\mathrm{mmol} / \mathrm{L})$. Most patients with bacterial meningitis have concentrations above $3.5 \mathrm{mmol} / \mathrm{L}$, with some values $>11 \mathrm{mmol} / \mathrm{L}(100 \mathrm{mg} / \mathrm{dL}) \quad(13$, 20-22, 31).

Increased CSF lactate is associated with low CSF glucose concentrations. While a moderate increase in lactate is often observed with normal CSF glucose, the occurrence of a very low CSF glucose concentration is usually associated with a substantial increase in lactate concentrations. This is indicative of increased anaerobic glycolysis by the adjacent cerebral tissue, or by cellular infiltrates in the leptomeninges (1).

The measurement of CSF lactate has been considered by some investigators to be clinically useful in the differential diagnosis of the disorders associated with low CSF glucose concentrations, and as a guide to the response to therapy (34).

The blood brain barrier (BBB) shows low permeability to the diffusion of lactate ions. At normal $\mathrm{pH}$, lactate is almost completely dissociated. The penetration of acid radicals of metabolic origin through the BBB is dependent on the characteristics of the exchange mechanisms (35).

In this study that compared patients with viral meningitis to the other groups (non-infectious CNS diseases, CSF normal, chronic meningitis and untreated bacterial meningitis), CSF lactate specificity and PPV were low. Although, when we compare only the two groups with acute meningitis, bacterial and viral, the sensitivity and specificity of CSF lactate for discriminating acute bacterial meningitis from viral meningitis is similar to results from previous studies. The PPV and NPV are also high.

A laboratory test that is easy to perform with highsensitivity is needed to identify patients with bacterial meningitis upon admission. Delayed treatment of false negative cases may be fatal. However, high specificity is desired to exclude expensive treatment of false positive cases (8). Previous studies have shown CSF lactate to have the highest sensitivity $(89 \%-100 \%)$ and specificity $(96 \%-100 \%)$ for discriminating acute untreated bacterial meningitis from viral meningitis, when the threshold limit was set at $3.5 \mathrm{mmol} / \mathrm{L}(36,37)$. This limit proved to also be relevant for tuberculosis (38), Listeria monocytogenes (39) and fungal meningitis (40). A higher discrimination limit of $>5.0 \mathrm{mmol} / \mathrm{L}$ diminished diagnostic sensitivity, while a lower limit of $2.8 \mathrm{mmol} / \mathrm{L}$ decreased diagnostic specificity and did not allow to distinguish viral meningitis from controls (12).

CSF glucose is considered by clinicians to be a good marker to discriminate acute bacterial meningitis from viral meningitis. However, some authors reported low-sensitivity (around $50 \%$ ) and high specificity with CSF glucose $(36,37,41)$. Because CSF glucose concentrations are dependent on blood glucose concentrations, blood must be collected at the same time as CSF. In contrast, CSF lactate concentrations are independent of blood concentrations (1). Thus, hyperglycemia and hypoglycemia may impair the CSF/blood glucose ratio, which exhibited $70 \%-91 \%$ sensitivity and $84 \%-99 \%$ specificity at a limit of $<0.4$ $(36,37)$.

Some authors suggest that despite the high-sensitivity and PPV of CSF lactate concentrations for the diagnosis of bacterial meningitis, results are generally non-specific and provide little additional diagnostic information. There is substantial evidence that whenever cerebral glycolysis is increased due to causes such as anaerobic glycolysis, hypoxia, ischemia, seizures or meningitis (42), lactate and pyruvate concentrations in brain and CSF and the lactate-to-pyruvate ratio are increased. However, measurement of CSF lactate concentrations was found to be superior to the ratio of CSF to blood glucose for the diagnosis of bacterial meningitis in postoperative neurological patients $(9,43)$. In partially treated bacterial meningitis, CSF lactate at a decision limit of $>3.2 \mathrm{mmol} / \mathrm{L}$ classified $88 \%$ of treated bacterial meningitis patients with $83 \%$ sensitivity and $91 \%$ specificity $(12)$ at a decision limit of $>3.5 \mathrm{mmol} / \mathrm{L}$, CSF lactate sensitivity decreased to $72 \%(36)$. 
In conclusion, CSF LA can be used as a routine test in laboratories. It is fast, automatized and easy to perform. It is a useful test for discriminating bacterial from viral meningitis with high sensitivity, specificity and predictive value. CSF LA can be helpful even in cases that cause diagnostic confusion, such as CSF samples suspect for viral meningitis, but with a predominance of neutrophils and normal glucose. Further work is needed in this area.

\section{References}

1. Fishman RA. Cerebrospinal fluid in diseases of the nervous system. Philadelphia: Saunders, 1992;431 pp.

2. Ponka A, Ojala K, Teppo AM, Weber TH. The differential diagnosis of bacterial and aseptic meningitis using cerebrospinal fluid laboratory tests. Infection 1983;11: 129-31.

3. Schut ES, de Gans J, van de Beek D. Communityacquired bacterial meningitis in adults. Pract Neurol 2008;8:8-23.

4. van de Beek D, de Gans J, Tunkel AR, Wijdicks EF. Community-acquired bacterial meningitis in adults. $\mathrm{N}$ Engl J Med 2006;354:44-53.

5. Quagliarello VJ, Scheld WM. Treatment of bacterial meningitis. N Engl J Med 1997;336:708-16.

6. Schlech WF. The epidemiology of bacterial meningitis. Antibiot Chemother 1992;45:5-17.

7. Baringer JR. Viral infections. In: Asbury AK, McKhann GM, McDonald WI, editors. Diseases of the nervous system: clinical neurobiology, 2nd ed. W.B. Saunders, Philadelphia 1992;1298-311.

8. Deisenhammer F, Bartos A, Egg R, Gilhus NE, Giovannoni G, Rauer S, et al. EFNS task force. Guidelines on routine cerebrospinal fluid analysis. Report from an EFNS task force. Eur J Neurol 2006;13:913-22.

9. Leib SL, Boscacci R, Gratzl O, Zimmerli W. Predictive value of cerebrospinal fluid (CSF) lactate versus CSF/blood glucose ratio for the diagnosis of bacterial meningitis following neurosurgery. Clin Infec Dis 1999;29:69-74.

10. Cabeça HLS, Gomes HR, Machado LR, Livramento JA. Dosage of lactate in the cerebrospinal fluid in infectious diseases of the central nervous system. Arq Neuropsiquiatr 2001;59:843-8.

11. Hutchesson A, Preece MA, Gray G, Green A. Measurement of lactate in cerebrospinal fluid in investigation of inherited metabolic disease. Clin Chem 1997;43:158-61.

12. Kleine TO, Zwerenz P, Zofel P, Shiratori K. New and old diagnostic markers of meningitis in cerebrospinal fluid (CSF). Brain Research Bull 2003;61:287-97.

13. Posner JB, Plum F. Independence of blood and cerebrospinal fluid lactate. Arch Neurol 1967;16:492-6.

14. Cunha BA. The usefulness of CSF lactic acid levels in central nervous system infections with decreased CSF glucose. Clin Infect Dis 2004;38:1260-1.

15. Pozo F, Tenório A. Detection and typing of lympgotropic herpesviruses by multiplex polymerase chain reaction. $J$ Virol Meth 1999;79:9-19.

16. Galen RS, Gambino SR. Beyond normality: the predictive value and efficiency of medical diagnoses. New York: John Wiley and Sons, 1975;237 p.

17. Steiner I, Budka H, Chaudhuri A, Koskiniemi M, Sainio K, Salonen O, et al. Viral encephalitis: a review of diagnostic methods and guidelines for management. Eur J Neurol 2005;12:331-43.

18. Tyler KL. Update on herpes simplex encephalitis. Rev Neurol Dis 2004;1:169-78.
19. Levinson A. Cerebrospinal fluid in health and disease. 3rd ed. C.V. Mosby: St Louis, 1929.

20. Brook I, Bricknell KS, Overturf GD, Finegold SM. Measurement of lactic acid in cerebrospinal fluid of patients with infections of the central nervous system. J Infect Dis 1978;137:384-90.

21. D'Souza E, Mandal BK, Hooper J, Parker L. Lactic acid concentration in cerebrospinal fluid and differential diagnosis of meningitis. Lancet 1978;2:579-80.

22. Kormorowski RA, Farmer SG, Hanson GA, Hause LL. Cerebrospinal fluid lactic acid in diagnosis of meningitis. J Clin Microbiol 1978;8:89-92.

23. Donald PR, Malan C. Cerebrospinal fluid lactate and lactate dehydrogenase activity in the rapid diagnosis of bacterial meningitis. S Afr Med J 1986;69:39-42.

24. Gastrin B, Briem H, Rombo L. Rapid diagnosis of meningitis with use of selected clinical data and gas liquid chromatographic determination of lactate concentration in cerebrospinal fluid. J Infect Dis 1979;139:529-33.

25. Knight JA, Dudek SM, Haymond RE. Early (chemical) diagnosis of bacterial meningitis-cerebrospinal fluid glucose, lactate, and lactate dehydrogenase compared. Clin Chem 1981;27:1431-4.

26. Nelson N, Eeg-Olofsson O, Larsson L, Ohman S. The diagnostic and predictive value of cerebrospinal fluid lactate in children with meningitis: its relation to current diagnostic methods. Acta Paediatr Scand 1986;75:52-7.

27. Spanos A, Harrell Jr FE, Durack DT. Differential diagnosis of acute meningitis: an analysis of the predictive value of initial observations. J Am Med Assoc 1989;262: 2700-7.

28. Controni G, Rodriguez WJ, Hicks JM, Ficke M, Ross S, Friedman G, et al. Cerebrospinal fluid lactic acid levels in meningitis. J Pediatr 1977;91:379-84.

29. Jordan GW, Statland B, Halsted C. CSF lactate in diseases of the CNS. Arch Intern Med 1984;143:85-7.

30. Zunt JR, Marra C. Cerebrospinal fluid testing for the diagnosis of central nervous system infection. Neurologic Clinic 1999;17:675-90.

31. Zupping R, Kaasick AE, Ravdam E. Cerebrospinal fluid metabolic acidosis and brain oxygen supply. Studies in patients with brain infarction. Arch Neurol 1971;23:33-8.

32. Yao H, Sadoshima S, Nishimura Y, Fujii K, Oshima M, Ishitsuka $T$, et al. Cerebrospinal fluid lactate in patients with diabetic mellitus and hypoglycemic coma. J Neurol Neurosurg Psychiatry 1989;52:372-5.

33. Gladden LB. Lactate metabolism: a new paradigm for the third millennium. J Physiol 2004;558:5-30.

34. Brook I. The importance of lactic acid in body fluids in the detection of of bacterial infections. Rev Infect Dis 1981;3:470-8.

35. Weyne J, van Leuven F. Lactic acid in the brain and cerebrospinal fluid in different conditions of hyperlactacidemia. Arch Int Physiol Biochim 1973;81:925-30.

36. Briem H. Comparison between cerebrospinal fluid concentrations of glucose, total protein, chloride, lactate, and total amino acids for differential diagnosis of patients with meningitis. Scand J Infect Dis 1983;15: 277-84.

37. Lindquist L, Linn T, Hansson LO, Kalin M, Axelsson G. Value of cerebrospinal fluid analysis in the diferential diagnosisd of meningitis: a study of 710 patients with suspected central system infection. Eur J Clin Microbiol Infect Dis 1988;7:374-80.

38. Tang LM. Serial lactate determinations in tuberculous meningitis. Scand J Infect Dis 1988;20:81-3.

39. Cunha $A B$, Fatehpuria $R$, Eisenstein LE. Listeria monocytogenes encephalitis mimicking Herpes simples encephalitis: the differential diagnostic importance of 
cerebrospinal fluid lactic acid levels. Heart Lung 2007; 36:226-31.

40. Body BA, Oneson RH, Herold DA. Use of cerebrospinal fluid lactic acid concentration in diagnosis of fungal meningitis. Ann Clin Lab Sci 1987;17:429-34.

41. Negrini B, Kelleher KJ, Wald ER. Cerebrospinal fluid findings in aseptic versus bacterial meningitis. Pediatrics 2000;105:316-9.
42. Tunkel AR, Hartman BJ, Kaplan SL, Kaufman BA, Roos $\mathrm{KL}$, Scheld WM, et al. Practice guidelines for the management of bacterial meningitis. Clin Infect Dis 2004; 39:1267-84.

43. Wong GK, Poon WS, Ip M. Use of ventricular cerebrospinal fluid lactate measurement to diagnose cerebrospinal fluid infection in patients with intraventricular haemorrhage. J Clin Neurosc 2008;15:654-5. 